\title{
Regulated Polyelectrolyte Nanogels for Enzyme Encapsulation and Activation
}

Ying Cai, ${ }^{\dagger}$ Peng Ding, ${ }^{*}+$ Jiaying Ni, ${ }^{\dagger}$ Lu Zhou, ${ }^{\star}$ Ayyaz Ahmad,,${ }^{\star}$ Xuhong Guo, ${ }^{\dagger}$ Martien A. Cohen Stuart, ${ }^{\dagger}$ Junyou Wang $^{*},+$

$\dagger$ Shanghai Key Laboratory of Multiphase Materials Chemical Engineering, East China University of Science and Technology, 130 Meilong Road, 200237, Shanghai, People's Republic of China

* Department of Chemical Engineering, MNS University of Engineering and Technology, Multan 60000, Pakistan 


\section{This PDF file includes:}

Figure S1. ${ }^{1} \mathrm{H}-\mathrm{NMR}$ spectrum of monomer and template.

Figure S2. ${ }^{1} \mathrm{H}-\mathrm{NMR}$ spectrum of before-purified and after-purified nanogels.

Figure S3. TEM images of PDMAEMA nanogels with different size and cross-link degree.

Figure S4. Cryo-TEM images of PDMAEMA nanogels (35 nm, 30\% MBA).

Figure S5. Relative Activity of free lipase, lipase@NG, and lipase@NG with additional PDMAEMA nanogel. 
Figure S1

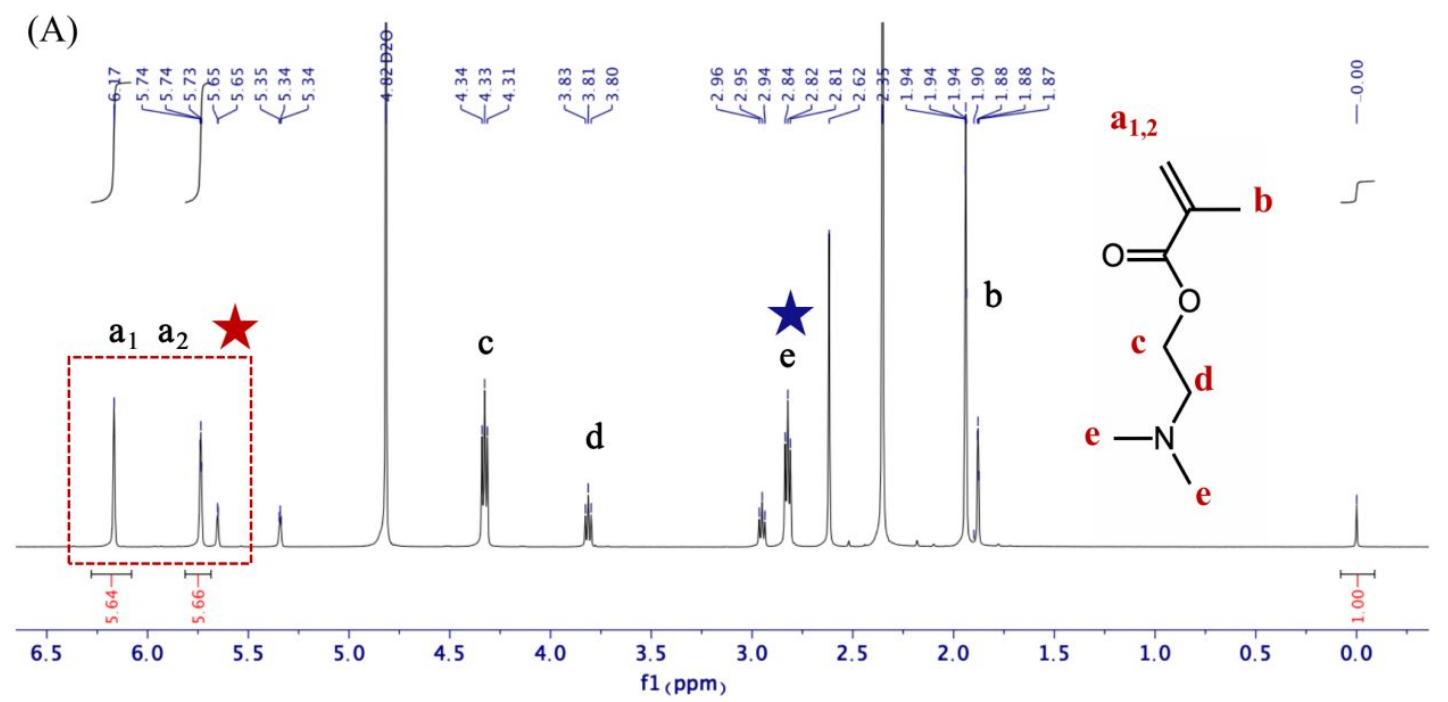

(B)

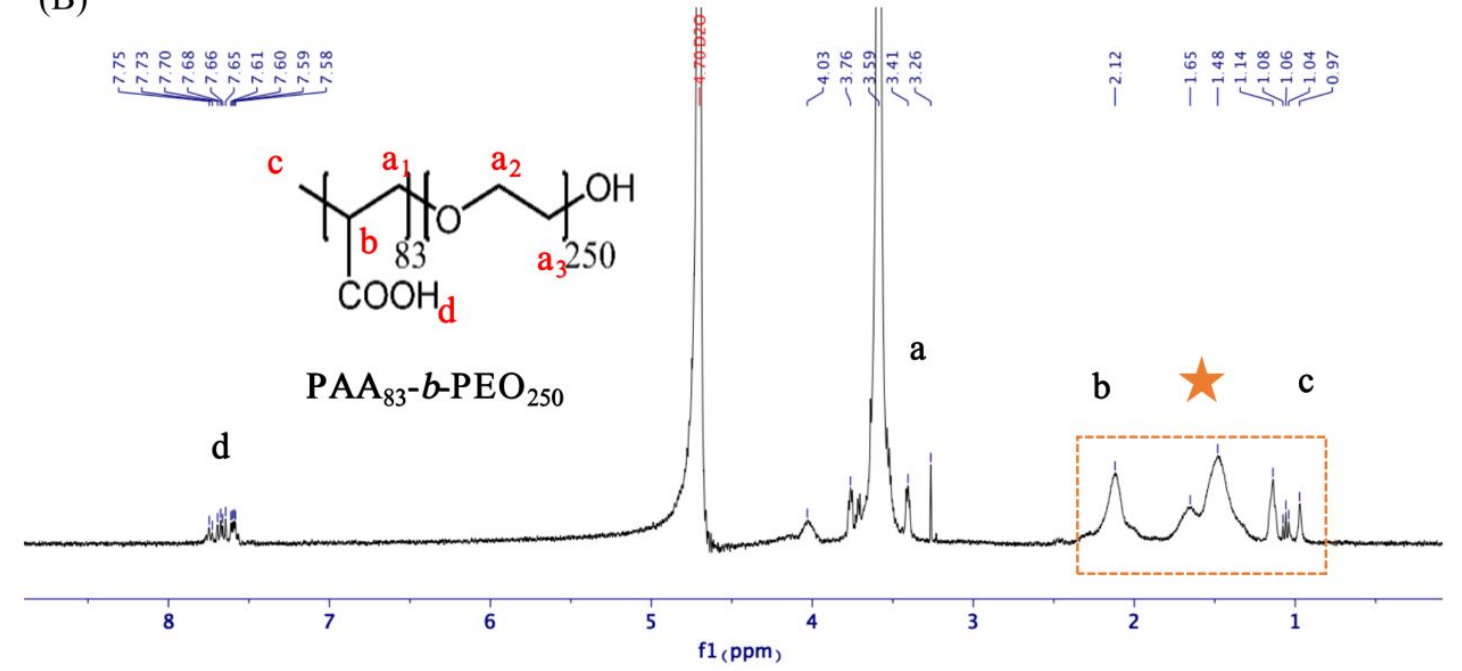

Figure S1. ${ }^{1} \mathrm{H}-\mathrm{NMR}$ spectrum of (A) DMAEMA and (B) $\mathrm{PAA}_{83}-b-\mathrm{PEO}_{250}$ template. Samples were dissolved in $\mathrm{D}_{2} \mathrm{O}(\mathrm{pH}$ was fixed at 7.0) and measured at $298 \mathrm{~K}$ on BRUKER AVANCE III 500 spectrometer (500 MHz).

As shown in Figure S1A, the chemical shift at 5.6-6.3 (red square), assigned to the carbon-carbon double bond of the DMAEMA monomer, is applied as the indicator for 
presence of the monomer. With 3-(Trimethylsilyl) propionic-2,2,3,3- $\mathrm{d}_{4}$ acid sodium salt (TSP) as an internal standard, the integral calculation is implemented to estimate the conversion of the monomers after polymerization. The chemical shift at 2.8 (blue start) is from $-\mathrm{CH}_{3}$ of the tertiary amine groups and is selected to notice the presence of PDMAEMA polymers in the nanogels. In Figure S1B, we select the peaks in the range of 0.8-2.4 (from the backbone of the polymers, specified by orange square and star,) as the indicator of $\mathrm{PAA}_{83}-b-\mathrm{PEO}_{250}$ template. 
Figure S2

(A)

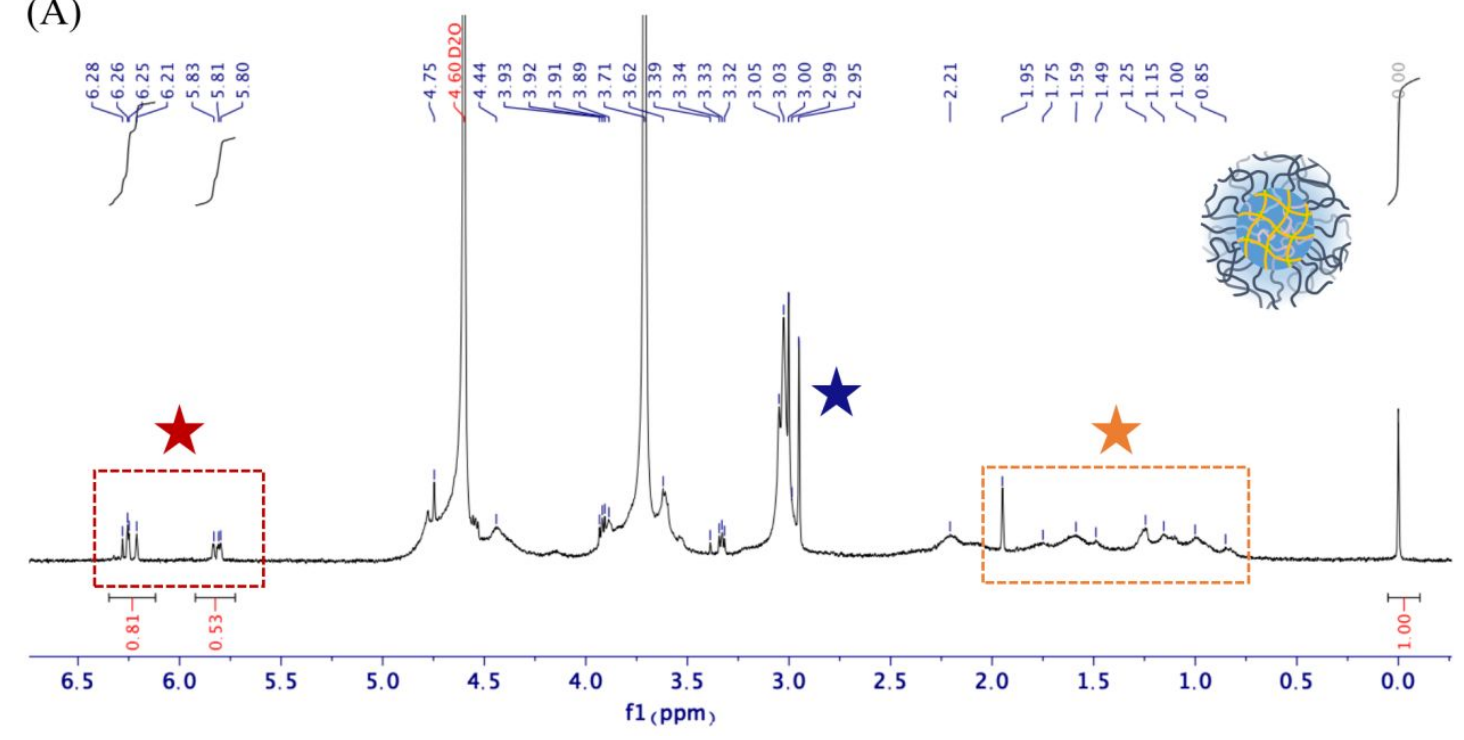

(B)

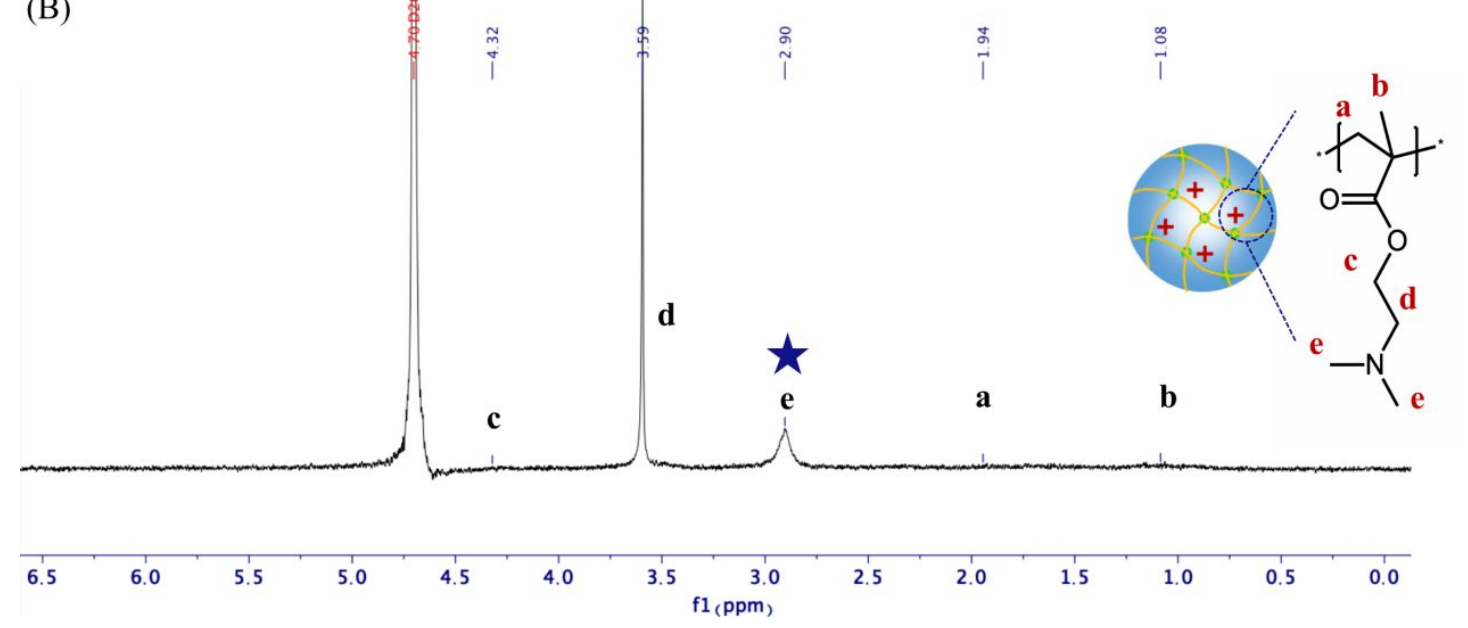

Figure S2. (A) ${ }^{1} \mathrm{H}-\mathrm{NMR}$ spectrum of the mixture after polymerization and PDMAEMA nanogels (B) after purification. Samples were dissolved in $\mathrm{D}_{2} \mathrm{O}(\mathrm{pH}$ was fixed at 7.0) and measured at $298 \mathrm{~K}$ on BRUKER AVANCE III 500 spectrometer (500 MHz). 
Figure S2A suggests that, about $10 \%$ of the DMAEMA monomer (red star) is remained in the mixture after the polymerization, which implies a $90 \%$ conversion of the monomers. Both the PDMAEMA (blue star) and template polymers (orange star) are present in the mixture before purification. While after centrifugation, only PDMAMEA chains remain in the nanogels, as evidenced by the typical chemical shift of PDMAEMA. These findings are consistent with our previous results, ${ }^{1}$ and confirm the successful polymerization and formation of PDMAEMA nanogels. The regulated structure and size of nanogels are further analyzed by static/dynamic light scattering and TEM, which are discussed in the main text. 


\section{Figure S3}
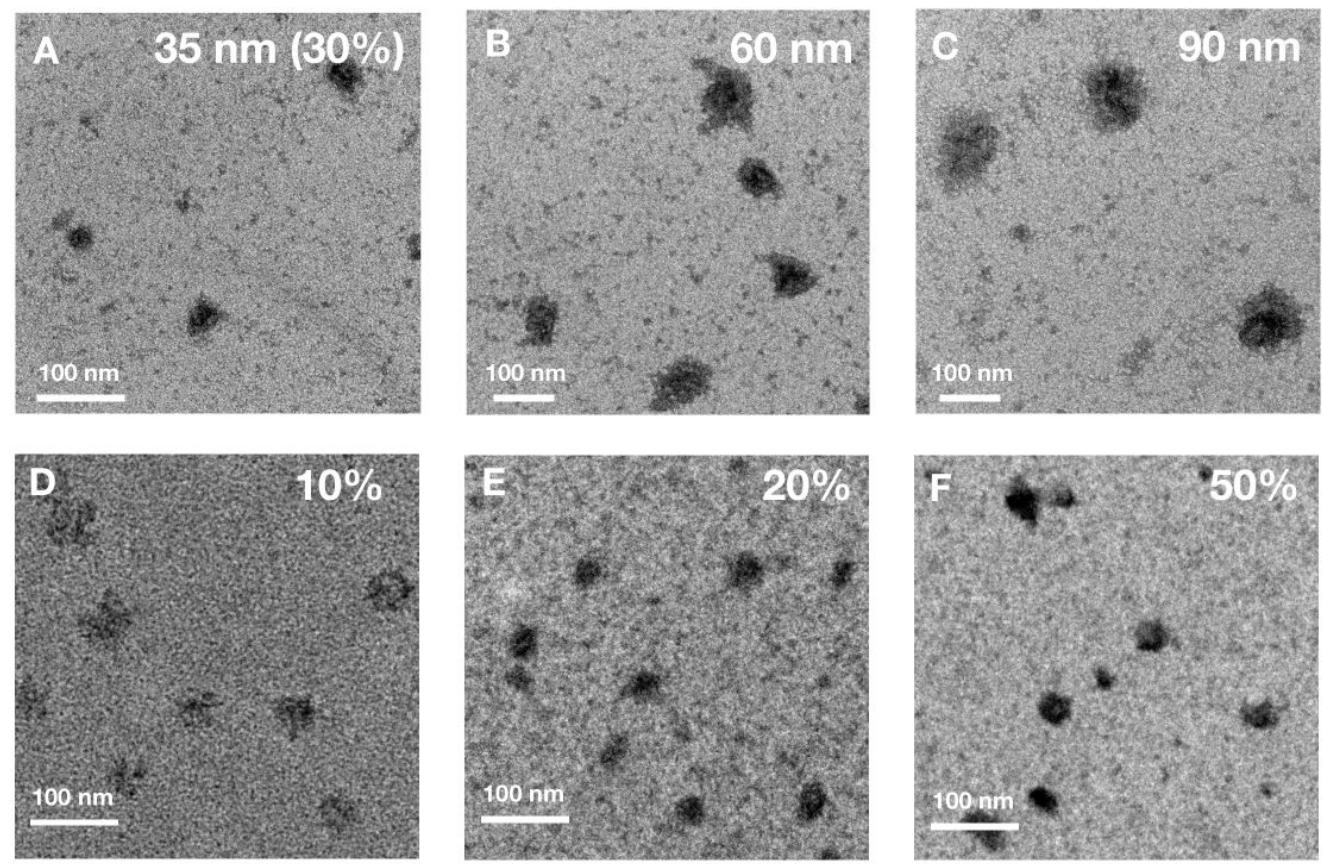

Figure S3. TEM images of PDMAEMA nanogels with different size and cross-link degree. A-C: nanogels with hydrodynamic radius of 35, 60 and $90 \mathrm{~nm}$ under fixed $30 \%$ MBA cross-linker. D-F: PDMAEMA nanogels prepared with $10 \%, 20 \%$ and 50\% MBA cross-linker ( $\mathrm{pH}$ was fixed at 7.0).

Figure S4

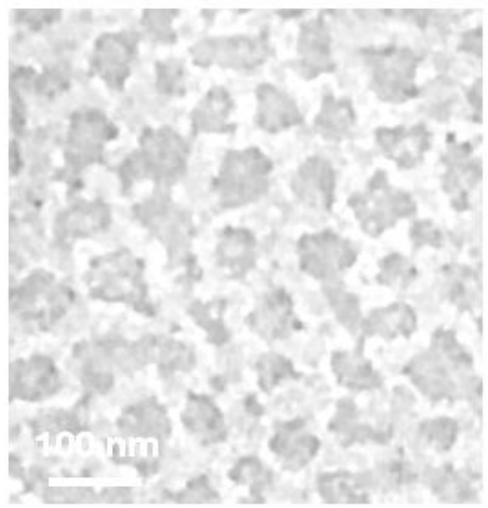


Figure S4. Cryo-TEM images of PDMAEMA nanogels with $30 \mathrm{~nm}$ size and $30 \%$ cross-link degree ( $\mathrm{pH}$ was fixed at 7.0). 
Figure S5

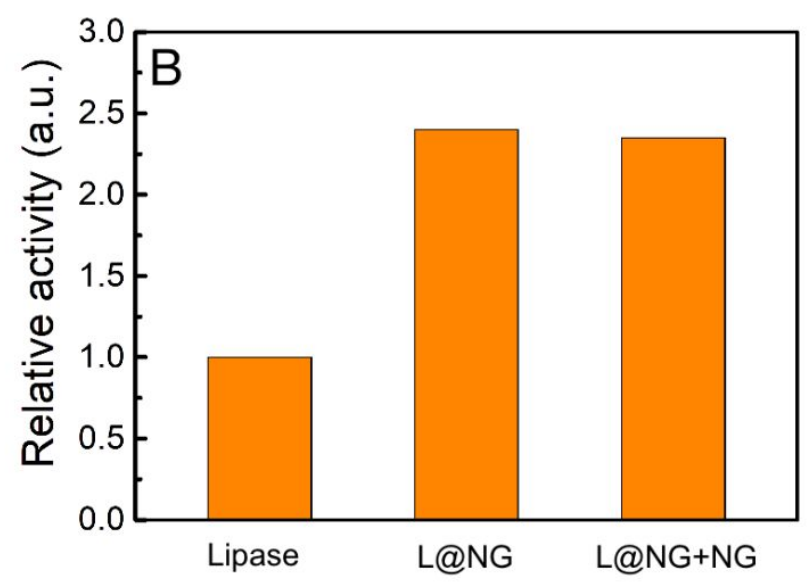

Figure S5. Relative Activity of free lipase, lipase@NG, and lipase@NG with additional PDMAEMA nanogel. 


\section{REFERENCE:}

1.Ding, P.; Huang, J.; Wei, C.; Liu, W.; Zhou, W.; Wang, J.; Wang, M.; Guo, X.; Cohen Stuart, M. A.;Wang, J. Efficient and Generic Preparation of Diverse Polyelectrolyte Nanogels by Electrostatic Assembly Directed Polymerization. CCS Chem. 2020, 2 (6), 1016-1025. 